\title{
Myeloproliferative Neoplasm with Prominent Eosinophilia
}

\author{
S GITI ${ }^{\mathrm{a}}$, M N Z BHUIYAN ${ }^{\mathrm{b}}$, M R HOSSAIN $^{\mathrm{c}}$, M S ISLAM ${ }^{\mathrm{d}}$, F AHMED ${ }^{\mathrm{e}}$ \\ M F HAQUEf ${ }^{\mathrm{f}}$, M M RAHMAN ${ }^{\mathrm{h}}$
}

\section{Summary:}

The patient, a young soldier aged 36 years having past history of malaria, was admitted in CMH Dhaka on 17 August 2011 as a transferred case from CMH Saidpur and died on 22 August 2011. The deceased was admitted in CMH Saidpur on 05 August 2011 with high fever for 05 days along with generalized joint and muscle pain. In spite of all available treatment the patient was deteriorating and he was then transferred to CMH Dhaka. At that time the patient was febrile, dehydrated and toxic with lymphadenopathy, extremely tender joints and muscles. The patient rapidly developed acute kidney failure and gradually developed

\section{Introduction:}

Eosinophilia is commonly observed in a wide range of non-clonal and clonal disorders. In the majority of cases it is reactive associated with atopic conditions, autoimmune disorders, infections or malignancies. In rare cases, a haematologic disorder underlies sustained eosinophilia which can be either non-clonal or clonal. Idiopathic hypereosinophilic syndrome (HES) and chronic eosinophilic leukemia (CEL) comprise a spectrum of indolent to aggressive disease chracterized by unexplained, persistent hypereosinophilia. Myeloproliferative and lymphoid neoplasms associated

a. Col Susane Giti, MBBS, MCPS, FCPS (Haematology), Dept. of Haematology, Armed Forces Institute of Pathology (AFIP), Dhaka Cantonment, Bangladesh.

b. Maj Mohammed Nuruzzaman Bhuiyan, MBBS, MCPS (Cl. Path), DCP, Dept. of Haematology, Armed Forces Institute of Pathology (AFIP), Dhaka Cantonment, Bangladesh.

c. Maj Gen Muhammad Rabiul Hossain, MBBS, MCPS, FCPS, FRCP, Consultant Physician, Directorate General of Medical Services (DGMS), Dhaka Cantonment, Bangladesh.

d. Maj Gen Md Saiful Islam, MBBS, MCPS, FCPS (Biochemistry), Commandant, Armed Forces Institute of Pathology (AFIP), Dhaka Cantonment, Bangladesh.

e. Brig Gen Faruk Ahmed, MBBS, MCPS, FCPS (Haematology), Deputy Commandant, Armed Forces Institute of Pathology (AFIP), Dhaka Cantonment, Bangladesh.

f. Brig Gen Md Fazlul Haque, MBBS, FCPS (Medicine), Chief Physician, Combined Military Hospital (CMH), Dhaka Cantonment, Bangladesh.

g. Lt Col Md Mizanur Rahman, MBBS, DCP, MCPS, FCPS (Haematology), Dept. of Blood Transfusion, Armed Forces Institute of Pathology (AFIP), Dhaka Cantonment, Bangladesh.

Address of Correspondence: Col Susane Giti, MBBS, MOPS, FCPS (Haematology), Dept. of Haematology, Armed Forces Institute of Pathology (AFIP), Dhaka Cantonment, Bangladesh, email: giti517@yahoo.com

Received: 10 July, 2012

Accepted: 2 February, 2013 features of DIC. His bone marrow examination revealed dyserythropoiesis with predominantly eosinophilic granulopoiesis, suggestive of myeloproliferative neoplasm with prominent eosinophilia. The patient was treated with injectable antibiotics, antimalarial and oral prednisolone with all intensive care facilities. Ultimately all attempts were proved unsuccessful and he died on 22 August 2011 at 1700 hrs. On autopsy the deceased had intra atrial thrombus and possibly that was the immediate cause of death. A haematological malignancy, myeloproliferative neoplasm with prominent eosinophilia, can very well produce such a fatal condition.

(J Banagladesh Coll Phys Surg 2013; 31: 162-167)

with rearrangement of platelet-derived growth factor receptor $\alpha$ (PDGFR $\alpha$ ), platelet-derived growth factor receptor $\beta$ (PDGFR $\beta$ ), fibroblast growth factor receptor 1 (FGFR1) are three rare specific disease groups, which have some shared features and some that differ. Clonal or primary eosinophilia is generally associated with chronic myeloproliferative disorders (Eos-MPD). Chronic eosinophilic leukemia (CEL) is diagnosed in the presence of increased numbers of blasts and/or clonality through cytogenetic or molecular analyses. ${ }^{1,2}$

\section{Case Report:}

The deceased, a young soldier of 36 years, was admitted to CMH Saidpur on 05 August 2011 with high fever for 05 days along with generalized joint and muscle pain. His urine R/E revealed 18-20 pus cells/HPF and widal test was positive (TO1:160). He was treated with sufficient dosage of inj Ceftriaxone and Doxycycline. Because of severe pain he was also given Diclofenac orally. During this time his serum albumin was found to be $17 \mathrm{gm} / \mathrm{L}$ and there was bilateral small renal calculi without any feature of obstruction. His urine culture was negative. His fever and pain continued, Ofloxacin and Quinine was added in the place of ceftriaxone (patient served in CHT and had malaria in the past). Inspite of all the above, the patient was deteriorating and he was then transferred to $\mathrm{CMH}$ Dhaka. The patient was febrile, dehydrated and toxic with extremely tender joints and muscles. There were palpable lymphnodes at the inguinal and cervical areas and mild hepatomegaly. Sufficient rehydration was done, Ofloxacin continued and oral prednisolone was advised considering a patient of polymyositis. His PBF report revealed Leucoerythroblastic blood picture with thrombocytopenia (Total count was $30 \times 10^{9} / \mathrm{L}, \mathrm{N}-78 \%$, E-14\% Myelocyte $2 \%$ with 
few promyelocyte and platelet count was $91 \times 10^{9} / \mathrm{L}$ ). Along with steroid his antibiotics was adjusted with inj Meropenem and Inj. Clarithromycin. Inspite of every thing, the patient rapidly developed acute kidney injury with serum creatinine of $6.5 \mathrm{mg} / \mathrm{dl}$ and metabolic acidosis with $\mathrm{Ph}$ of 7.2 . Patient become unconscious and also developed generalized seizure. He was given assisted ventilation and peritoneal dialysis was done. He was also given Inj Albumin and combination of anti malarial (Artemether+ Lumefantrine). Gradually he developed features of DIC (Bilirubin 2-5 mg/dl, ALT-30 U/L, Alk phosphatse -82 U/L, LDH-3649 mg/dl, PT-20 (control12), APTT-45 (control 30), FDP $>5 \mu \mathrm{mol} / \mathrm{L}$ and thrombocytopenia). His bone marrow examination revealed dyserythro-poiesis with predominantly eosinophilic granulopoiesis, suggestive of myeloproliferative neoplasm with prominent eosinophilia. Ultimately all attempts were proved unsuccessful and he died on 22 August 2011 at 1700 hrs.

Postmortem examination revealed a mural thrombus in the right atrium. Considering all the above facts, the deceased had following important features. a. High fever, polyarthritis and tender painful muscles. b. Lymphadenopathy. c. Pyrexia. d. Hypoalbuminaemia, raised TO in widal test, subsequently he also had: e. Rapid development of AKI and acidosis. f. Leucoerythroblastic blood picture with thrombocytopenia , eosinophilia and bone marrow evidence of MPN with prominent eosinophilia. Chronic eosinophilic leukaemia and HES is excluded by the presence of $<5 \%$ blasts in bone marrow and eosinophilia associated with features of myeloproliferative syndrome ${ }^{3}$ (including organomegaly neutrophilia, circulating myelocytes and erythroblasts, hyperplastic bone marrow) respectively. g. Features of DIC. h. Right atrial thrombus.

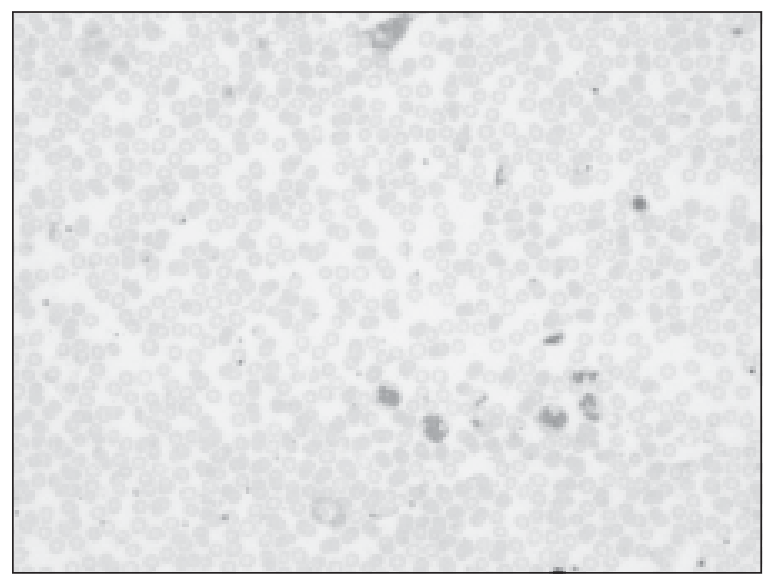

Fig.-1: Peripheral blood film.

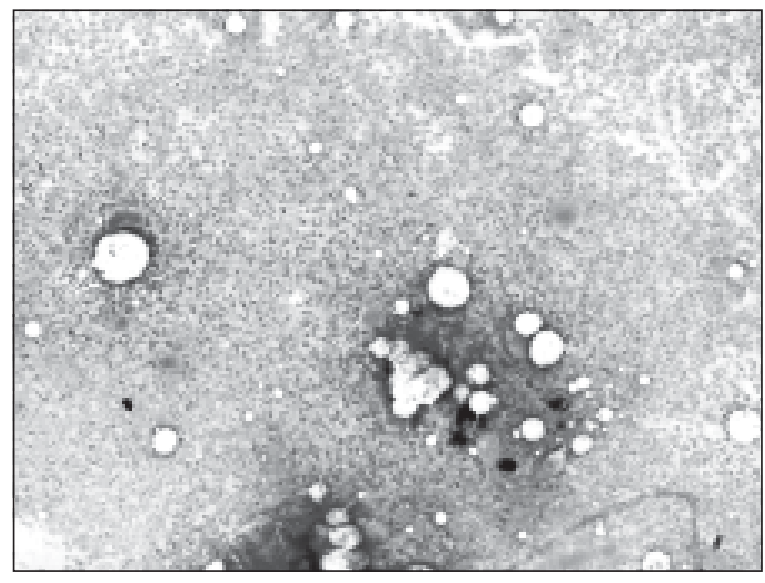

Fig.-2: Bone marrow (Low power view)

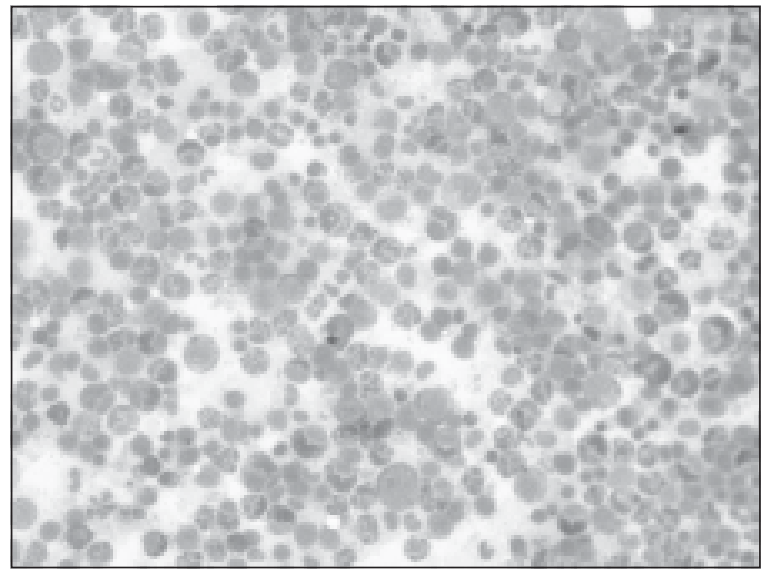

Fig.-3: Bone marrow (High power view)

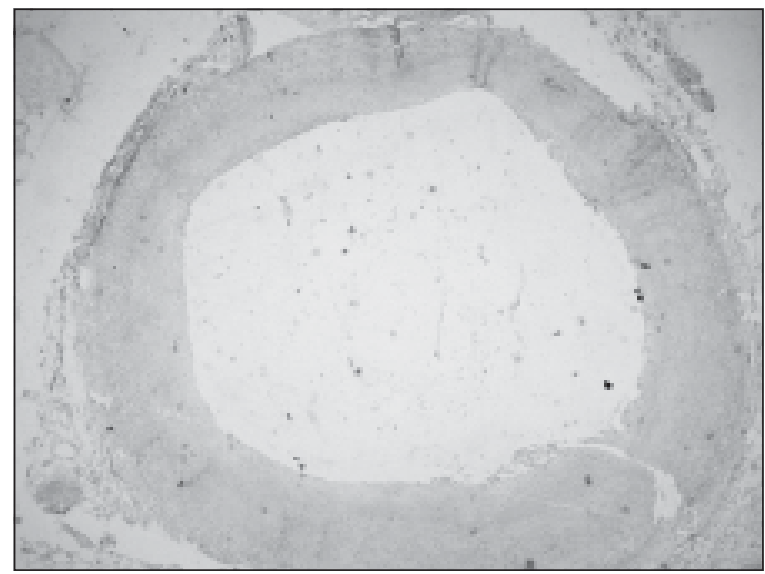

Fig.-4: Intact coronary artery (Microscopic view) 


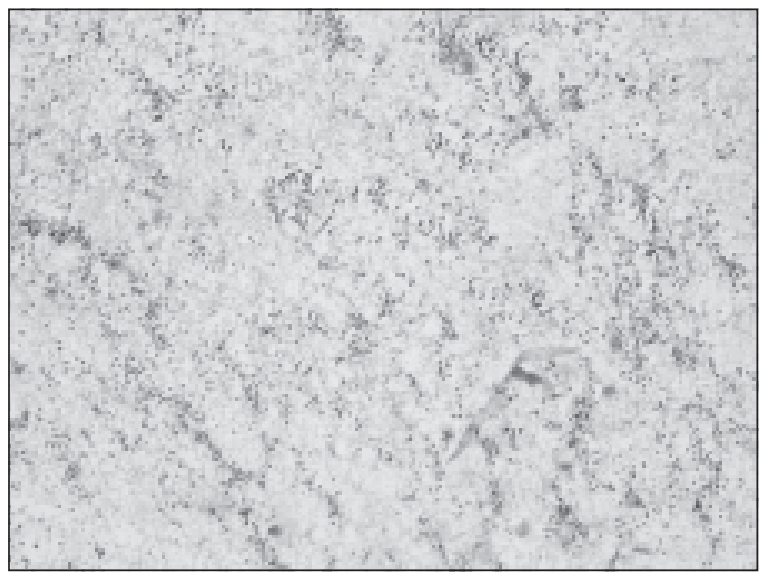

Fig.-5: Infected mural thrombus (Low power view)

At CMH Saidpur, after admission and subsequently also, the deceased had persistent hypoalbuminaemia. There is no obvious cause about this very important marker of chronic illness, in that there is no history of malnutrition, jaundice or renal problem in the past. It could be due to presence of an indolent haematological malignancy which was sub-clinical or due to a connective tissue disease (Polymyositis). After admission there was pyuria, though sufficient antibiotic was given, in a immunocompromised state, UTI can cause septicaemia and ultimately DIC. The deceased had intra atrial thrombus and possibly that was the immediate cause of death. Presence of thrombus indicates a Pro-coagulant state. A haematological malignancy can very well produce such a condition. So finally considering all the evidences above, the mode of death was due to cardiac arrest form intra-atrial thrombus. The primary cause of which was a haematological malignancy i.e Myeloproliferative neoplasm with prominent eosinophilia.

\section{Discussion:}

The 2008 World Health Organization (WHO) classification of tumors of haematopoietic and lymphoid tissues introduced a new category for myeloid and lymphoid neoplasms with eosinophilia and abnormalities of PDGFR $\alpha$, PDGFR $\beta$ or FGFR1. Many of these cases present as a myeloproliferative neoplasm, usually with eosinophilia. However, neoplasms associated with rearrangement of PDGFR $\alpha$ can present as acute myeloid leukemia or as $\mathrm{T}$ lymphoblastic lymphoma with eosinophilia. Neoplasms associated with rearrangement of FGFR1 even more frequently present as acute myeloid leukemia or $\mathrm{T}$ lymphoblastic lymphoma, in both instances

with eosinophilia, and both $\mathrm{T}$ lymphoblastic and $\mathrm{B}$ lymphoblastic transformation of chronic eosinophilic leukemia have also been described. Because of the prominent lymphoid component these disorders have been assigned, in the WHO classification, to a specific category rather than being categorized as a myeloproliferative neoplasm (MPN). However, it should be noted that BCR- ABL1-positive chronic myelogenous leukemia is accepted as a bonafide myeloproliferative neoplasm and yet it too can undergo lymphoblastic transformation and even present as acute lymphoblastic leukemia with the underlying chronic myelogenous leukemia being revealed only after remission has been achieved. In the case of PDGFRBrelated disease, the features of MPN are more variable but are often those of chronic myelomonocytic leukaemia (CMML) with eosinophilia ${ }^{4}$.

Chusid et al in 1975 used 3 diagnostic criteria for HES that are still utilized today: (1) persistent eosinophilia of $1.5 \times 10^{9} / \mathrm{L}\left(1500 / \mathrm{mm}^{3}\right)$ for longer than 6 months; (2) lack of evidence for parasitic, allergic, or other known causes of eosinophilia;and (3) signs and symptoms of organ involvement ${ }^{5}$. In the recent World Health Organization (WHO) classification, a diagnosis of HES or CEL requires exclusion of reactive causes of eosinophilia and malignancies. In this scheme, blood eosinophilia is divided into 3 categories: reactive (nonclonal eosinophilia), clonal disorders of the bone marrow associated with eosinophilia, and HES, which remains a diagnosis of exclusion ${ }^{6}$.

Acquired constitutive activation of protein tyrosine kinases is a central feature in the pathogenesis of chronic MPD. Activation occurs as a consequence of specific point mutations, e.g. JAK2 V617F, or fusion genes, e.g. BCR-ABL, generated by chromosomal translocations, insertions or deletions. In Eos-MPD, cytogenetic analysis has identified four distinct recurrent breakpoint clusters that target the genes encoding PDGFR $\alpha$ at 4q12, PDGFR $\beta$ at 5q31-33, FGFR1 at 8p11 and janus kinase 2 (JAK2) at 9p24 ${ }^{7}$. To date, more than 35 different fusion genes have been identified in association with EosMPD, the most common of which is FIP1L1-PDGFRA, generated by a cytogenetically invisible $800 \mathrm{~kb}$ interstitial deletion on chromosome 4q12 ${ }^{8}$. 


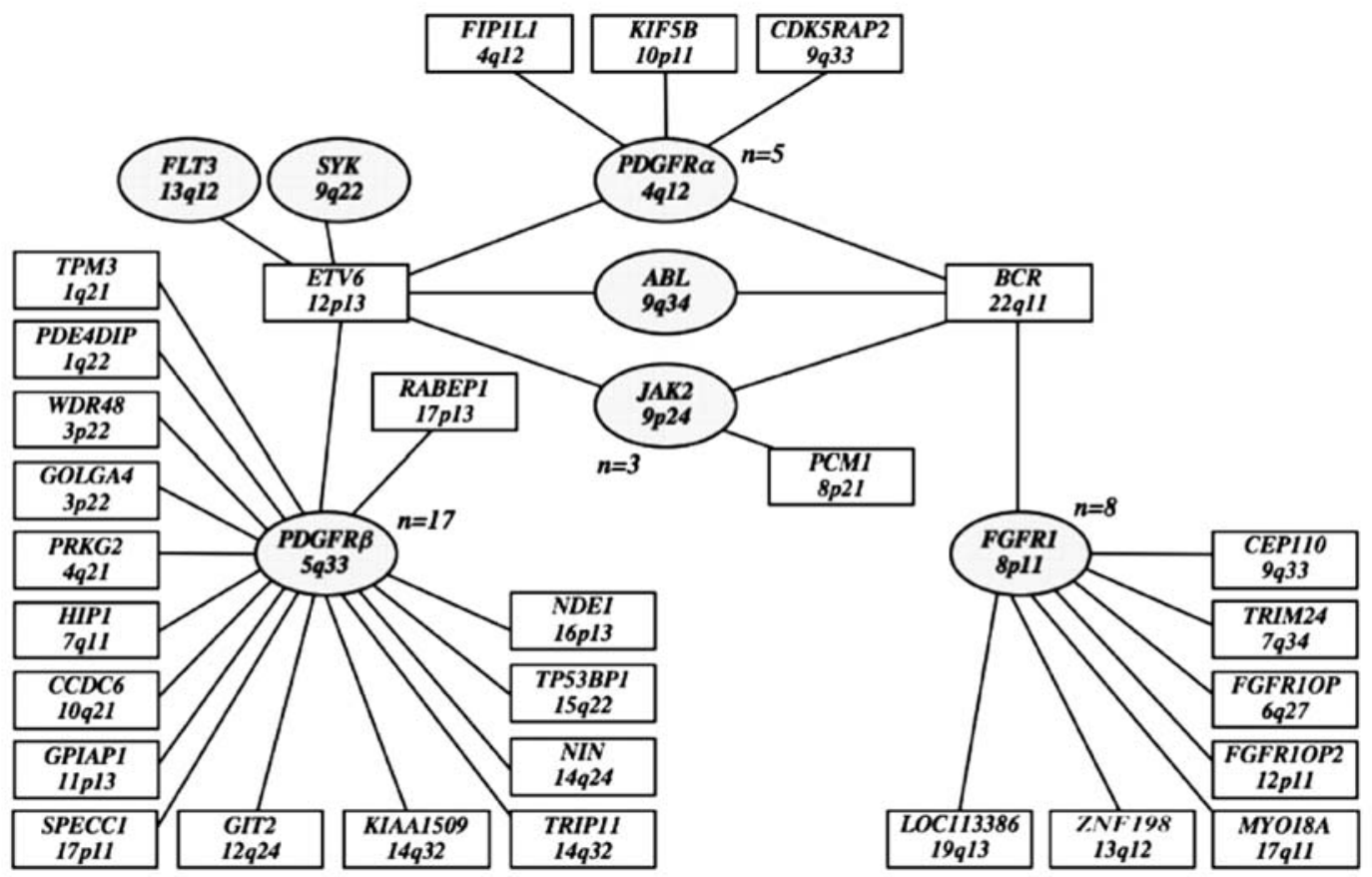

Fig.-6: Molecular pathogenesis

Clonal or primary eosinophilia is generally associated with chronic myeloproliferative disorders (Eos-MPD) which is a potentially life-threatening condition associated with end-organ damage to heart, gastrointestinal tract, skin, joints or nervous system due to release of granular contents from infiltrating eosinophils. The most common presenting signs and symptoms were weakness and fatigue (26\%), cough (24\%), dyspnea (16\%), myalgias or angioedema (14\%), rash or fever (12\%), and rhinitis (10\%). In many cases lymphadenopathy is present at diagnosis whereas in others it appears during the course of the disease. The most striking clinical feature, similar to PDGFR-related disorders, is a marked male predominance. The majority of patients have organ and systemic involvement like blood, heart, spleen, liver, lung, nervous system and especially, skin involvement ${ }^{9,10,11}$.

The most serious clinical findings relate to endomyocardial fibrosis, with ensuing restrictive cardiomegaly. Scarring of the mitral/tricuspid valves leads to valvular regurgitation and formation of intracardiac thrombi, which may embolize to the brain or elsewhere. Peripheral neuropathy, central nervous system dysfunction, pulmonary symptoms due to lung infiltration, and rheumatological findings are other frequent manifestations ${ }^{4}$.

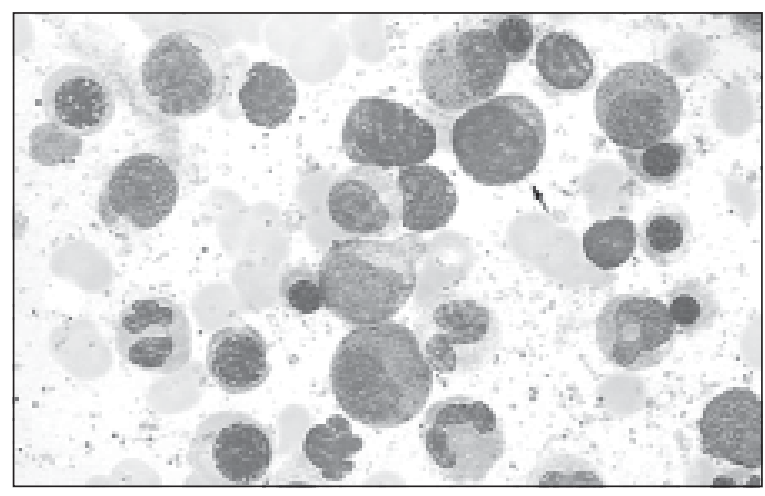

Fig.-7: Bone marrow (High power view-book picture).

The effectiveness of imatinib for myeloproliferative HES has revolutionized treatment of this disease. Many patients can be managed utilizing relatively low doses of imatinib, 100-200 mg/week, with complete clinical and molecular control. Before commencing treatment with imatinib, troponin should be measured because of the danger of eosinophilic myocarditis and treatment with glucocorticoids may be required. Cases of primary resistance to imatinib has also been identified. Treatment of patients with myeloproliferative HES lacking FIP1L1PDGFRA can present a considerable challenge, and one must resort to the medications including prednisolone, hydroxycarbamide and interferon-á. A monoclonal antibody to IL-5, mepolizumab, is also used ${ }^{12,13}$. 


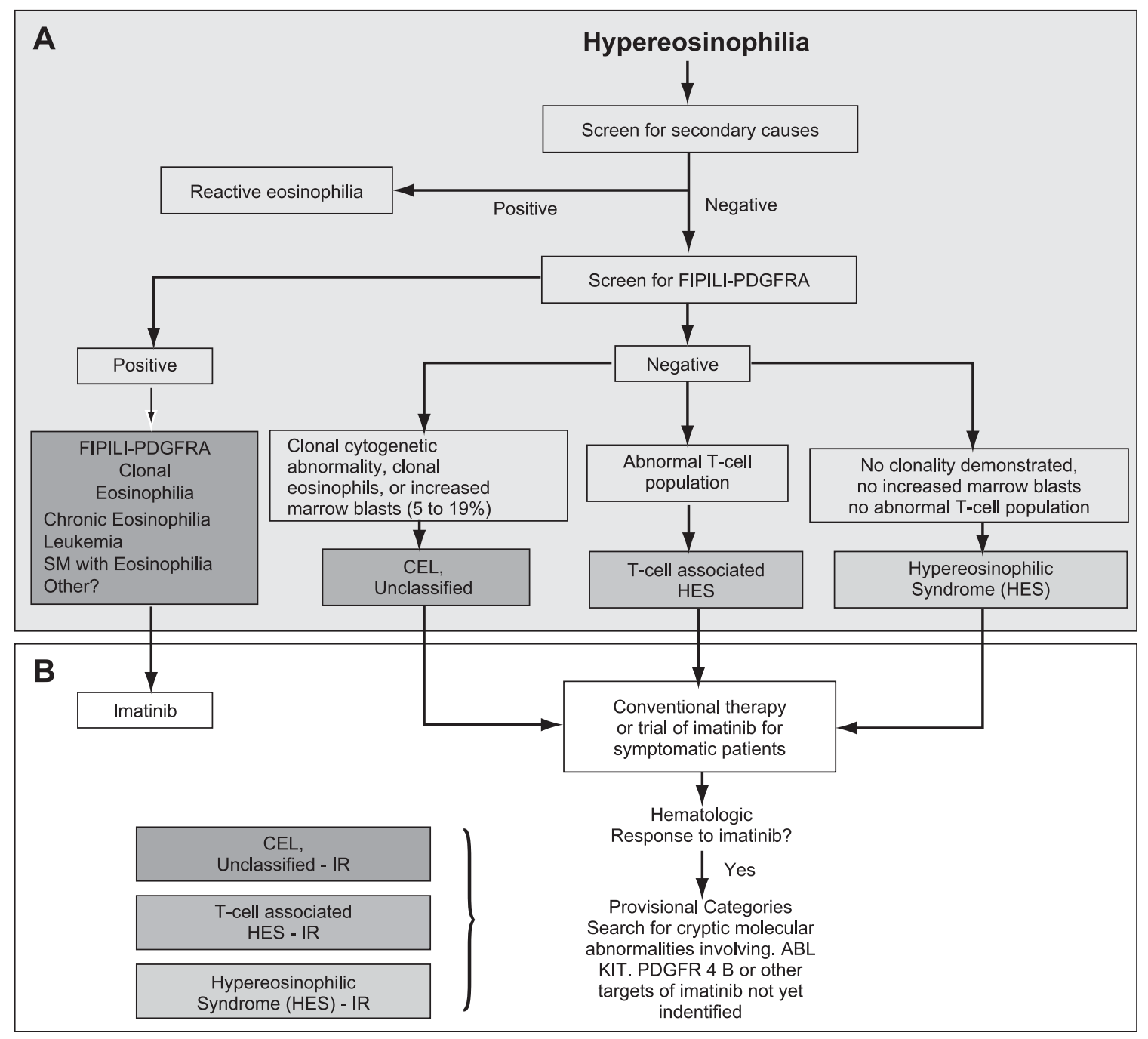

Fig.-8: Possible diagnostic and treatment algorithm for hypereosinophilia.

\section{Conclusion:}

Myeloproliferative and lymphoid neoplasms associated with rearrangement of PDGFR $\alpha$, PDGFR $\beta$ and FGFR1 constitute three rare specific disease groups, in whom eosinophilia is characteristics. The identification of FIP1L1-PDGFR $\alpha$ in cases of HES adds to a growing list of activated fusion tyrosine kinases linked to the pathogenesis of chronic myeloproliferative disorders. It is unique but the relative rarity of this molecular genetic analysis would represent a considerable challenge. However, circumstantial evidences could be gained to address this condition. The overall prognosis is currently poor. In fact, this discussion points to the rapid pace of progress in the understanding of diagnosis and management including several kinase inhibitors, are on the horizon, to treat myeloproliferative neoplasm with prominent eosinophilia.

\section{References:}

1. Tefferi A, Patnaik MM, Pardanani A. Eosinophilia: secondary, clonal and idiopathic. Br J Haematol 2006;133:468-92.

2. Gotlib J, Cross NC, Gilliland DG. Eosinophilic disorders: molecular pathogenesis, new classification, and modern therapy. Best Pract Res Clin Haematol 2006;19:535-69.

3. Ogbogu PO, Bochner BS, Butterfield JH, et al. Hypereosinophilic syndromes: a multicenter retrospective analysis of clinical characteristics and response to therapy. J Allergy Clin Immunol 2009; 124 (6):1319-1325.

4. Bain BJ, Gilliland DG, Horny H-P, Vardiman JW. Myeloid and lymphoid neoplasms with eosinophilia and abnormalities 
of PDGFRA, PDGFRB and FGFR1. In: Swerdlow SH, Campo E, Harris NL, Jaffe ES, Pileri SA, Stein H, Thiele J and Vardiman JW (eds) World Health Organization Classification of Tumours of Haematopoietic and Lymphoid Tissue, 4th edn. 2008 IARC Press, Lyon, pp. 68-73.

5. Chusid MJ, Dale DC, West BC, Wolff SM. The hypereosinophilic syndrome. Analysis of fourteen cases with review of the literature. Medicine. 1975;54:1-27.

6. Bain B, Pierre R, Imbert M, Vardiman JW, Brunning RD, Flandrin G. Chronic eosinophilic leukaemia and the hypereosinophilic syndrome. In: Jaffe ES, Harris NL, Stein H, Vardiman JW, eds. World Health Organization of Tumours: Tumours of Haematopoietic and Lymphoid Tissues. Lyon, France: IARC Press; 2001:29-31.Chusid

7. Reiter A, Walz C, Cross NCP. Tyrosine kinases as therapeutic targets in BCR-ABL negative chronic myeloproliferative disorders. Current Drug Targets 2007;8:205-16.
8. Griffin JH, Leung J, Bruner RJ, Caligiuri MA, Briesewitz R. Discovery of a fusion kinase in EOL-1 cells and idiopathic hypereosinophilic syndrome. Proc Natl Acad Sci USA 2003;100:7830-5.

9. Brito-Babapulle F. Clonal eosinophilic disorders and the hypereosinophilic syndrome. Blood Rev. 1997;11:129-145.

10. Lefebvre C, Bletry O, Degoulet P, et al. Prognostic factors of hypereosinophilic syndrome. Study of 40 cases. Ann Med Interne (Paris). 1989;140: 253-257.

11. Weller PF, Bubley GJ. The idiopathic hypereosinophilic syndrome. Blood. 1994;83:2759-2779.

12. Jovanovic JV, Score J, Waghorn K, Cilloni D, Gottardi E, Metzgeroth G, et al. Low-dose imatinib mesylate leads to rapid induction of major molecular responses and achievement of complete molecular remission in FIP1L1-PDGFRA positive chronic eosinophilic leukemia. Blood 2007;109:4635-41.

13. Krause DS, Van Etten RA. Tyrosine kinases as targets for cancer therapy. N Engl J Med 2005;353:172-87. 